\title{
Genitourinary Symptoms Associated with Chlamydia trachomatis and Neisseria gonorrhoeae Infections in a Tertiary Care Hospital in Oman
}

\author{
Zakariya Al-Muharrmi, ${ }^{1}$ "Richard Lau, ${ }^{2}$ Ahad Al-Balushi, ${ }^{1}$ Alyazi Al-Saadi, ${ }^{1}$ Zeyana Al-Habsi, ${ }^{3}$ Ali Elgalib, ${ }^{3}$ \\ Samir Shah, ${ }^{3}$ Maha Al-Fouri, ${ }^{3}$ Bader Al-Rawahi, ${ }^{3}$ Seif Al-Abri ${ }^{4}$
}

ABSTRACT: Objectives: This study aimed to determine the pattern of clinical presentations associated with Chlamydia trachomatis and Neisseria gonorrhoeae infections. Methods: A retrospective cohort study was conducted between 2015 and 2020 and included patients attending Sultan Qaboos University Hospital, Muscat, Oman, who were tested for these infections using nucleic acid amplification. Results: A total of 408 women and 89 men were included. A total of 11 infections were identified in women $(n=11,2.7 \%)$ and 14 in men $(n=14,15.7 \%)$. Chlamydia accounted for almost all infections in women $(\mathrm{n}=10,90.9 \%)$, commonly presenting with lower abdominal pain or abnormal vaginal bleeding. In men, urethral discharge/dysuria syndrome was the most common presentation and chlamydia was identified in eight men and gonorrhoea in six; a majority of all infections were seen in patients $\leq 35$ years old. Conclusion: The relative prevalence of two common sexually transmitted infections (STIs) among patients with genitourinary symptoms at a tertiary referral hospital have been described. The pattern of their presentations will inform the design of prospective studies to improve surveillance and guide public health policy in Oman. This study highlights the need for a multi-sectoral approach involving all providers to enable comprehensive STI surveillance.

Keywords: Sexually Transmitted Diseases; Chlamydia trachomatis; Neisseria gonorrhoeae; Oman.

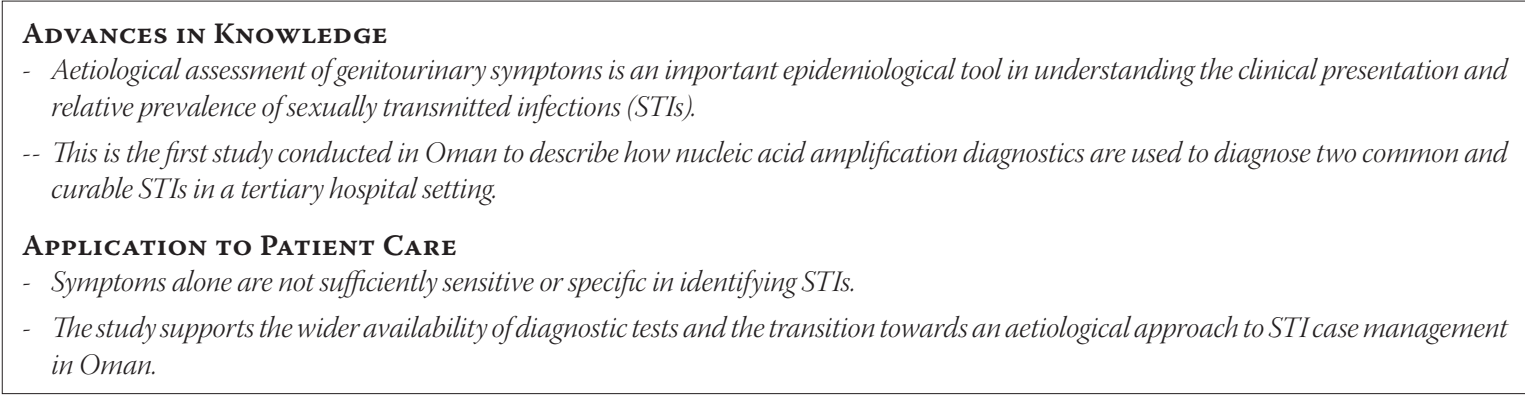

S EXUALly TRANSMITTED INFECTIONS (STIS) are an important focus of public health policy in Oman. A key aspect for STI control is the early and efficacious management of patients, using either a syndromic approach or clinical management based on laboratory tests (aetiological approach). The latter approach, especially when using highly accurate and sensitive molecular diagnostic tests, has the advantage of identifying the specific STI pathogen(s) so that targeted antimicrobial therapy can be administered, with the potential of improving STI case management and surveillance as well as avoiding unnecessary and wasteful treatments.

Oman's public health services have largely relied on the syndromic management approach, with the utilisation of flowcharts (algorithms) for each of the five recognised STI syndromes-male urethral discharge, vaginal discharge, lower abdominal (pelvic) pain in women, scrotal swelling and genital ulceration. ${ }^{1}$
Laboratory tests are not undertaken for most STI patients who receive syndromic management. Nevertheless, periodic laboratory-based aetiological assessments are necessary to check for the relevance of the flowcharts and laboratories play a key role in STI surveillance, research programmes and forming public health policies. ${ }^{2-4}$

In contrast to public health services, Sultan Qaboos University Hospital (SQUH), Muscat, Oman, has been providing STI diagnostics for the detection of Neisseria gonorrhoeae and Chlamydia trachomatis infections since 2014 for patients presenting with genitourinary symptoms, including infertility. These are two of the most common curable STIs and are associated with significant sexual and reproductive morbidity including adverse birth outcomes, pelvic inflammatory disease, tubal factor infertility, ectopic pregnancy and epididymo-orchitis. ${ }^{5-7}$ 
There are regional differences in the prevalence of chlamydia and gonorrhoea, with the Middle East and North Africa (MENA) region having relatively low rates of both infections in either gender compared to other regions, notably the Americas and Western Pacific region. ${ }^{5}$ While cultural influences and adherence to religious teachings may explain some of these differences in the MENA region, stigma, embarrassment, lack of awareness and limited access to molecular diagnostic tests may also account for under-reporting.

As the aetiology of STI syndromes in Oman has never previously been studied using molecular diagnostic tests, the purpose of this exploratory study was to investigate the relative prevalence of chlamydia and gonorrhoea associated with genitourinary symptoms to inform patient care and identify areas of STI surveillance that may require strengthening.

\section{Methods}

This was a retrospective cohort study of patients who attended SQUH and tested for gonorrhoea and chlamydia. SQUH is a 600-bed university teaching hospital and tertiary referral centre based in the Seeb wilayat (district) in the Muscat governorate, the capital of Oman.

The study population consisted of women who were tested between January and December 2020 and men who were tested between January 2015 and December 2020. The Xpert ${ }^{\circledR}$ CT/NG (Cepheid Inc., Sunnyvale, California, USA) nucleic acid amplification test (NAAT) was used for chlamydia and gonorrhoea detection from endocervical swabs (women) or firstcatch urine specimens (men). ${ }^{8}$ Women who were pregnant and patients who had taken antibiotics in the previous two weeks or had invalid NAAT results (from improper sample processing, polymerase chain reaction inhibition or sample processing control not being detected in the test sample) were excluded. Tests for HIV and syphilis were offered to patients diagnosed with chlamydia and gonorrhoea and to those requesting screening.

The test results were recorded and matched with the patients' sociodemographic information (age, gender, marital status), the type of clinic they attended and their main presenting symptom.

Descriptive statistics were used to describe the study population. The differences between groups were assessed using the Chi-square test. Significance was defined at as $P$ value of $\leq 0.05$ and a $95 \%$ confidence interval (CI) was calculated for a proportion using Wilson's method for small samples. ${ }^{9}$ Based on their clinical presentation, women were grouped into one of five categories: (1) lower abdominal (pelvic) pain; (2) abnormal vaginal bleeding (menorrhagia, irregular, inter- and post-coital bleeding); (3) abnormal vaginal discharge (malodour, increased volume, altered colour); (4) asymptomatic women attending for infertility assessment; or (5) women attending for contraception, pre-operative assessment and urogynaecological symptoms. Men were categorised into two groups-(1) those with urethral discharge/ dysuria syndrome or (2) those with other presentations including asymptomatic, scrotal or abdominal pain, haematuria, investigation of fever and unspecified presentations. The data collection process was affected due to clinical and laboratory resources being diverted to manage the on-going COVID-19 pandemic. These resource limitations meant that it was not possible to collect and collate more data for women presenting before 2020 .

The study was approved by the Medical Research Ethics Committee, College of Medicine and Health Sciences, SQUH (SQU-EC/210/19). All data collected for this study were anonymised and identifiable details were removed.

\section{Results}

A total of 416 women attended SQUH with genital or gynaecological symptoms including infertility in 2020 .

Table 1: Sociodemographic characteristics of patients attending Sultan Qaboos University Hospital who were tested for chlamydia and gonorrhoea in women in 2020 and in men between 2015 and $2020(\mathrm{~N}=497)$

\begin{tabular}{lcc} 
Characteristic & \multicolumn{2}{c}{$\mathbf{n}(\%)$} \\
Nationality & Female $(\mathbf{n}=408)$ & Male $(\mathbf{n}=89)$ \\
Omani & $394(96.6)$ & $84(94.4)$ \\
Non-Omani & $14(3.4)$ & $5(5.6)$ \\
Age distribution in years & \\
Mean & 36.5 & 33.8 \\
Median & 35.95 & 31.2 \\
Range & $16.5-66.7$ & $14.3-81.1$ \\
Marital status & & $39(43.8)$ \\
Married & $398(97.5)$ & $0(0)$ \\
Divorced & $5(1.2)$ & $0(0)$ \\
Widowed & $4(1.0)$ & $35(39.3)$ \\
Single & $1(0.2)$ & $15(16.9)$ \\
Undisclosed & $0(0)$ &
\end{tabular}


Table 2: Main symptom or presentation and detection of gonorrhoea and chlamydia in women and men $(\mathrm{N}=497)$

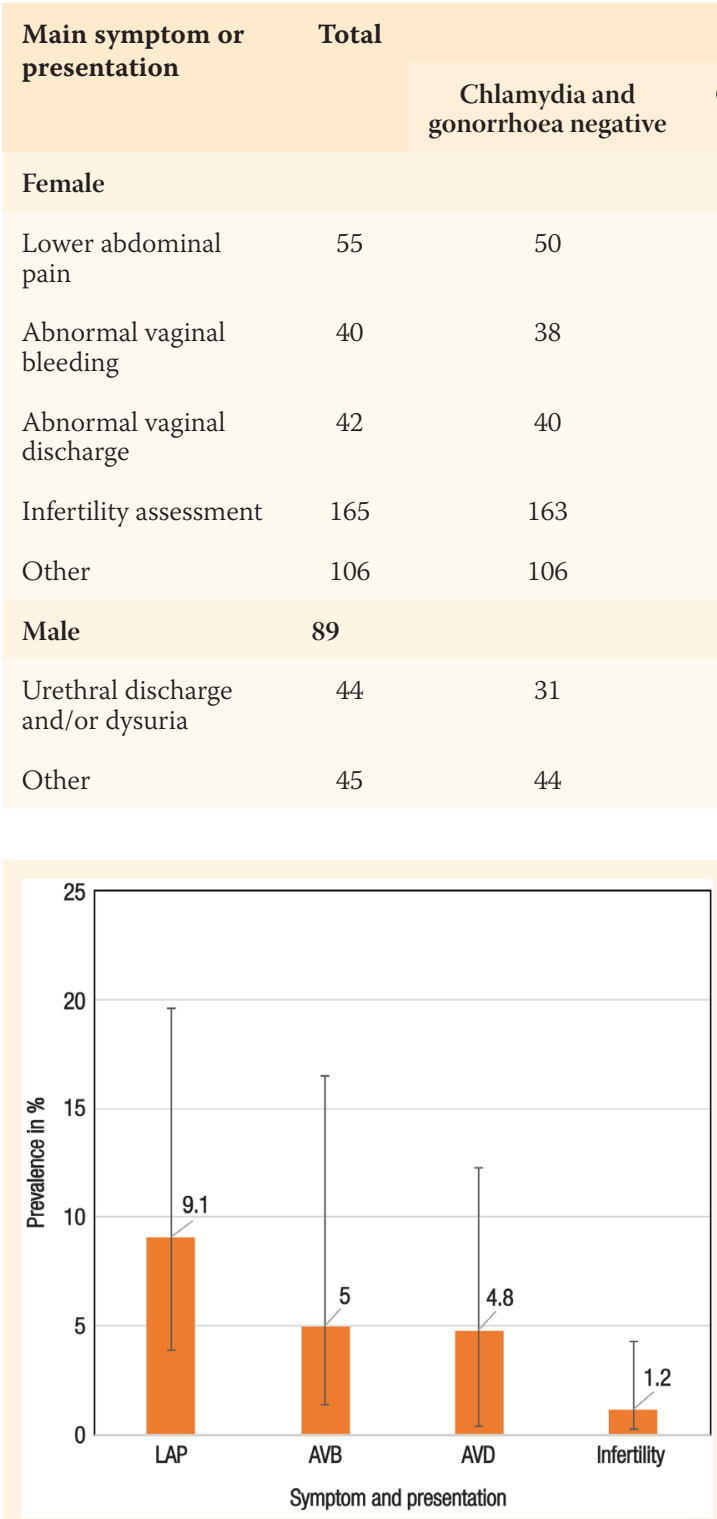

Figure 1: Prevalence of chlamydial infection in percentage with $95 \%$ confidence intervals in women presenting with lower abdominal pain, abnormal vaginal bleeding, abnormal vaginal discharge and infertility.

$L A P=$ abdominal pain; $A V B=$ abnormal vaginal bleeding; $A V D=$ abnormal vaginal discharge.

Eight women were excluded from the study because they were either pregnant $(n=5)$ or had invalid test results $(n=3)$; therefore, a total of 408 women were included in this study [Table 1].

The majority of the women $(\mathrm{n}=235,57.6 \%)$ attended the gynaecology clinic, some ( $\mathrm{n}=159,39.0 \%)$ attended the infertility clinic and the remainder $(\mathrm{n}=$ 14, 3.4\%) attended other clinical services (Accident and Emergency $=13$, General Medicine $=1$ ).

A total of $10(2.5 \%)$ women were infected with Chlamydia, nine (2.2\%) of whom were mono-infected and one woman dually infected with Chlamydia and gonorrhoea. One (0.25\%) woman was mono-infected n

Chlamydia positive Gonorrhoea positive Chlamydia and only only gonorrhoea positive 408

$\begin{array}{lll}4 & 0 & 1 \\ 2 & 0 & 0 \\ 1 & 1 & 0 \\ 2 & 0 & 0 \\ 0 & 0 & 0 \\ 8 & 5 & 0 \\ 0 & 1 & 0\end{array}$

with gonorrhoea. Lower abdominal (pelvic) pain and abnormal vaginal bleeding presentations accounted for most infections [Table 2]; however, symptoms alone were not sufficiently specific or sensitive in correlation with infection [Figure 1]. Women aged 26-35 years had the highest prevalence of chlamydial infection $(7 / 10,70 \%)$ [Figure 2]. More infections were identified in women $\leq 35$ years old than in those $>35$ years old (Chi-squared $=7.83 ; P=0.0051$ ).

Data on 99 men who attended SQUH and were tested for gonorrhoea and chlamydia between 2015 and 2020 were collected. Some patients were excluded $(\mathrm{n}=10)$ due to invalid NAAT test results. The data for the remaining 89 men were included and analysed [Table 1]. Most patients $(n=44,49.4 \%)$ presented with urethral discharge and/or dysuria syndrome. Chlamydia was detected in eight men (9.0\%) and gonorrhoea in six (6.7\%); there were no dual infections [Table 2]. Although tests on men were ordered from a wider range of clinics within SQUH, with most tests being ordered by Infectious Diseases $(n=22)$ and General Medicine $(\mathrm{n}=21)$ clinics, more infections were disproportionately identified in men attending the Accident and Emergency ( $n=2 / 3,67.0 \%)$, Student Health ( $\mathrm{n}=3 / 6,50.0 \%$ ) and Family Practice clinics ( $\mathrm{n}=$ 4/18, 22.0\%). Compared to women, infections appeared to occur in younger men more often [Figure 1]; however, group comparisons were not possible because of the modest sample size of men.

\section{Discussion}

This study describes the relative prevalence of two important, curable STIs in symptomatic patients 


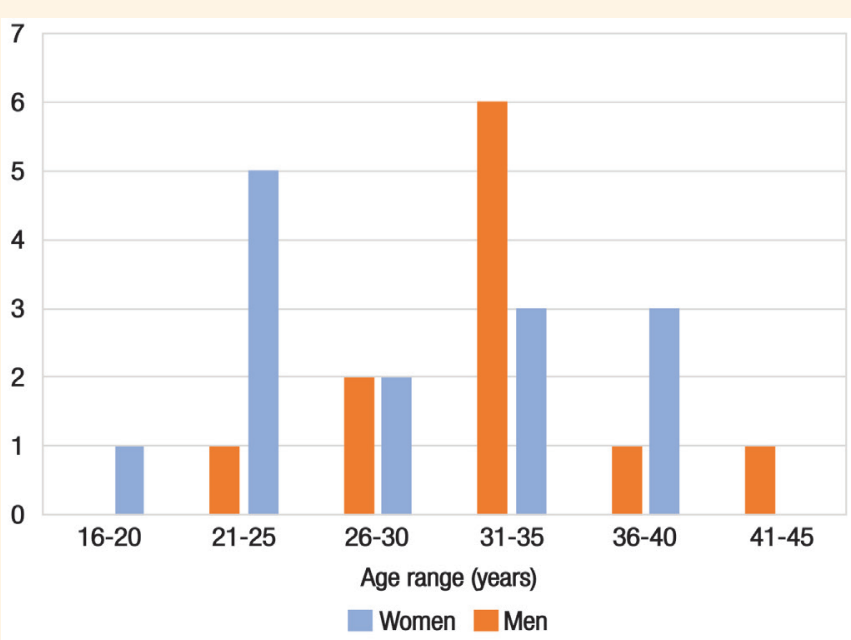

Figure 2: Distribution of chlamydia and gonorrhoea cases by age and gender.

presenting to a tertiary care hospital in Muscat, Oman. The most common presentation in symptomatic women was lower abdominal pain, a cardinal feature of pelvic inflammatory disease (PID) and associated with a $17 \%$ risk of infertility after one episode. ${ }^{10}$ Although the prevalence of infection was highest for this symptom in women, it was not significantly different from other presentations in this cohort, suggesting that symptoms alone are not sufficiently sensitive or specific to reliably identify an STI [Figure 2]. Given the significant caseload attributed to chlamydial infection, the study illustrates the importance of widening access to STI diagnostics beyond clinic-based syndromic case management to screen for infections before complications such as PID develop. The costeffectiveness of such a strategy will, however, depend on the local prevalence of these infections, how they present and the behavioural characteristics of the local population.

In the current female cohort, an age of $\leq 35$ years was identified as a significant STI risk factor; this was consistent with epidemiological studies that demonstrated a higher risk in younger women. ${ }^{11-13}$ However, age may also be a confounder linked to other social, cultural or behavioural factors such as duration of relationship, partner behaviour and condom use that will require further analysis in a prospective study.

The much higher prevalence of gonorrhoea in men compared to women (6.7\% versus $0.5 \%$ ) could be explained by the self-selection of men with more symptomatic infections, as male gonococcal urethritis tends to produce a more vigorous inflammatory response than corresponding gonococcal urethritis or cervicitis in women, which can often be asymptomatic or associated with non-specific symptoms. In contrast, the smaller size of the male cohort may disproportionately reflect more symptomatic men electing to seek acute care elsewhere (possibly private clinics) for reasons of confidentiality. The observations support the need for a prospective multi-centre study incorporating other providers of STI care and completion of an anonymised patient questionnaire to identify risk behaviours that may explain these differences.

The current study has several limitations. The patients included in the study were those that chose to seek healthcare for their symptoms from one provider (SQUH) in Muscat and may not be representative of patients living elsewhere or accessing other providers in the rest of the country/city. As with any retrospective study, there are also inherent biases that may hinder the applicability of the present findings to the wider population. Recording of particular symptoms (and not recording others) by the attending clinician could lead to selection bias. Misclassification bias may also have occurred when data were abstracted from the clinical records. A further consideration is that many different clinicians (especially for men) were involved in patient care; therefore, the recording of symptoms as risk factors may be less accurate than what could be achieved through a prospective cohort study.

\section{Conclusion}

This exploratory study provides insight into the relative prevalence of two common STIs and their presentations. The study findings support age-targeted, culturally appropriate prevention and education strategies as well as broadening the availability of STI diagnostic tests to detect asymptomatic STIs, especially in women. The study also highlights the need in Oman for a multi-sectoral approach involving all providers to enable comprehensive STI surveillance. 


\section{AUTHORS' CONTRIBUTIONS}

ZAM, RL and ZAH designed the study. AAB and AAS retrieved and collated clinical and laboratory data. All the authors were responsible for drafting and critical appraisal of the manuscript. All authors approved the final version of the manuscript.

\section{CONFLICT OF INTERESTS}

The authors declare no conflict of interests.

\section{FUNDING}

No funding was received for this study.

\section{References}

1. Alary M, Abreu H, Anupong C, Ballard R, Jakobsone I, Kahindo M, et al. Guidelines for the management of sexually transmitted infections. Geneva, Switzerland: WHO Publications, 2001. From: http://apps.who.int/iris/bitstream/handle/10665/42782/ 9241546263 _eng.pdf; j s es sion id=B 69 C C 0 E C CF43C5E8FA35C7B9748C82AE?sequence $=1$ Accessed: July 2021

2. Rietmeijer CA, Mungati M, Machica A, Mugurungi O, Kupara V, Rodgers L, et al. The etiology of male urethral discharge in Zimbabwe: Results from the Zimbabwe STI etiology study. Sex Transm Dis 2018; 45:56-60. https://doi.org/10.1097/ OLQ.0000000000000696.

3. Tadesse E, Teshome M, Amsalu A, Shimelis T. Genital Chlamydia trachomatis infection among women of reproductive age attending the gynaecology clinic of Hawassa University referral hospital, southern Ethiopia. PLoS One 2016; 11:e0168580. https://doi.org/10.1371/journal.pone.0168580.

4. Newman LA, Wi T, Sharkey L, Broutet N, Temmerman M, Toskin I. A tool for strengthening STI surveillance at the country level. Geneva, Switzerland: WHO Publications, 2015. From: https://apps.who.int/iris/handle/10665/161074 Accessed: Jul 2021
5. Newman LA, Rowley J, Vander Hoorn S, Wijesooriya NS, Unemo M, Low N, et al. Global estimates of the prevalence and incidence of four curable sexually transmitted infections in 2012 based on systematic review and global reporting. PLoS One 2015; 10:e0143304. https://doi.org/10.1371/journal. pone. 0143304

6. Reekie J, Donovan B, Guy R, Hocking JS, Kaldor JM, Mak D, et al. Risk of ectopic pregnancy and tubal infertility following gonorrhoea and chlamydia infections. Clin Infect Dis 2019; 69:1621-3. https://doi.org/10.1093/cid/ciz145.

7. Tang W, Mao J, Li KT, Walker JS, Chou R, Fu R, et al. Pregnancy and fertility-related adverse outcomes associated with Chlamydia trachomatis infection: A global systematic review and metaanalysis. Sex Transm Infect 2020; 96:322-9. https://doi. org/10.1136/sextrans-2019-053999.

8. Gaydos CA, Van Der Pol B, Jett-Goheen M, Barnes M, Quinn N, Clark C, et al. Performance of the Cepheid CT/NG Xpert rapid PCR test for detection of Chlamydia trachomatis and Neisseria gonorrhoeae. J Clin Microbiol 2013; 51:1666-72. https://doi. org/10.1128/JCM.03461-12.

9. Confidence limits for a proportion. From: https://epitools. ausvet.com.au/ciproportion Accessed: Jul 2021.

10. Price MJ, Ades AE, Soldan K, Welton NJ, Macleod J, Simms I, et al. The natural history of Chlamydia trachomatis infection in women: A multi-parameter evidence synthesis. Health Technol Assess 2016; 20:1-250. https://doi.org/10.3310/hta20220.

11. Holmes KK. The chlamydia epidemic. JAMA 1981;245:1718-23. https://doi.org/10.1001/jama.1981.03310420010002.

12. Adams EJ, Charlett A, Edmunds WJ, Hughes G. Chlamydia trachomatis in the United Kingdom: A systematic review and analysis of prevalence studies. Sex Transm Infect 2004; 80:354-62. https://doi.org/10.1136/sti.2003.005454.

13. Rowley J, Vander Hoorn S, Korenromp E, Low N, Unemo M, Abu-Raddad LJ, et al. Chlamydia, gonorrhoea, trichomoniasis and syphilis: Global prevalence and incidence estimates, 2016. Bull World Health Organ 2019; 97:548-62P. https://doi. org/10.2471/BLT.18.228486. 